\title{
Solving the relativistic rankine-hugoniot condition in presence of magnetic field in astrophysical scenario
}

\author{
Ritam Mallick \\ Department of Physics, Indian Institute of Science, Bangalore 560012, INDIA
}

\begin{abstract}
Rankine-Hugoniot condition has been solved to study phase transition in astrophysical scenario mainly in the case of phase transition from neutron star (NS) to quark star (QS). The phase transition is brought about by a combustion front, which travels from the center to the surface. The equations of state and temperature plays a huge role in determining the nature of the front propagation, which brings about the phase transition in neutron stars (NSs). Magnetic field has been introduced and the modified conservation condition for the perpendicular and oblique shocks is obtained. Numerical solution of the perpendicular shock has been shown in the figures, which finds that the magnetic field helps in shock generation. It indirectly hints at the instability of the matter and thereby the NS for very high magnetic field, implying that NSs can only support finite magnetic field strength.
\end{abstract}

Keywords: stars: neutron, stars: magnetic fields, equation of state

\section{Introduction}

When the velocity of a fluid in motion becomes comparable with or exceeds that of the sound, the effect due to compressibility of the fluid become of prime importance. For a wave propagating in a non conducting gas, when the amplitude is so small that linear theory applies, the disturbance propagates as a sound wave. If the gas has a uniform pressure and density, the speed of propagation of sound and the wave profile maintains a fixed shape, since each part of the wave moves with same speed. However, when the wave

Email address: ritam@physics.iisc.ernet.in (Ritam Mallick) 
possesses a finite amplitude, so that nonlinear terms in the equation become important, the crest of the sound wave moves faster than its leading or trailing edge. This causes a progressive steepening of the front portion of the wave as the crest catches up and ultimately, the gradient of pressure, density, temperature and velocity become large that dissipative processes, such as viscosity or thermal conduction are no longer negligible. Then a steady wave shape is attained, called a shock wave. The shock wave moves at a speed in excess of the sound speed, so the information cannot be propagated ahead to signal its imminent arrival, since such information would travel at sound speed relative to the undisturbed medium ahead of the shock. The dissipation inside the shock front leads to a gradual conversion of the energy being carried by the wave into heat. Thus, the effect of the passage of a shock wave are to convert ordered (flow) energy into random (thermal) energy through particle collisions and also to compress and heat the gas. The shock front itself is in reality a very thin transition region. Its width is typically only a few mean-free paths, with particle collisions establishing the new uniform state behind the shock.

The relativistic shock propagates into a medium with a changing equation of state. Therefore, a simple analysis of the jump condition for a polytropic or perfect fluid is not adequate and a deep understanding of this problem calls on for the full theoretical description of the relativistic shock in a medium with arbitrary equation of state. Further complication might arise if there is a presence of significant magnetic field. In fact, one can show that the relative importance of a magnetic field can grow during a collapse. In the field of nuclear physics, high energy collisions among heavy ions can be modeled by using fluid dynamical concepts. Also, some current models under investigation predict that relativistic shocks (or relativistic detonation and deflagration) might be related to the phase transition from nuclear matter to a quark matter.

Relativistic shock waves have been the subject of early investigation in relativistic fluid dynamics and magneto-fluid dynamics. In relativistic fluid dynamics the pioneering work is that of Taub [1] where the relativistic form of jump condition is established. A detailed analysis of the thermodynamic treatment of classical shock waves, is due to Thorne [2]. Explicit solutions of the jump condition have ben obtained for special equation of states. Shock wave in relativistic magneto-fluid have been investigated extensively and in rigorous mathematical way by Lichnerowicz. [3]. Detonation and deflagration waves in relativistic magneto-fluid dynamics for nuclear physics and 
cosmology have been investigated by [4, 5].

In Astrophysical scenario shock plays very important role in determining the outcome of compact stars. In the case of massive stars, in the range between 8-100 solar masses, which are thought to be the progenitors of type II supernovas, one of the most viable mechanism for producing a explosion is gravitational collapse and bounce [6]. In this case a shock is formed outside the inner core, which propagates outwards reaching relativistic speeds. Shock waves are also responsible for phase transition and gamma ray bursts (GRB) in compact stars.

In this paper I will mostly concentrate on the phase transition scenario in a compact star. Witten [7] conjectured of strange quark matter (SQM), consisting of approximately equal numbers of up $(u)$, down $(d)$ and strange $(s)$ quarks, is believed to be the ground state of strong interaction. This was supported by model calculations for certain ranges of values for strange quark mass and strong coupling constant [8]. After that there has been constant efforts at confirming the existence of and SQM, though transiently, in ultra relativistic collisions. On the other hand, SQM could naturally occur in the cores of compact stars, where central densities are expected to be an order of magnitude higher than the nuclear matter saturation density. Thus, neutron stars which have sufficient high central densities might convert to strange star, or at least hybrid (a star with a quark core) stars. These transitions may lead to various observable signatures in the form of a jump in the breaking index and gamma ray bursts [9, 10], and a full QS might help in explaining the phenomena of observed quasi periodic oscillations [11].

There may be several scenarios by which neutron stars could convert to quark stars. It may happen through a "seed" of external SQM [12], or triggered by the rise in the central density due to a sudden spin-down in older neutron stars [13]. Several authors have studied the conversion of nuclear matter to strange matter under different assumptions [14, 15, 16, 17, 18, 19, 20, 21, 22, 23, 24]. They have been summarized in a recent work of ours [25] and for the constraint of space, I do not repeat them here.

After the discovery magnetars, some compact stars were found to have very high surface magnetic fields. So the study of relativistic rankine-hugoniot condition is not sufficient. To have a full understanding of the properties of NS and its phase transition to QS, such conditions should be examined in the presence of high magnetic fields. In this paper I wish to carry out such a basic calculation keeping our focus mainly on the astrophysical scenario. The paper is organized as followed: first I will discuss the general rankine 
hugoniot condition as a discontinuity in the conversion front. In section III, I will introduce magnetic field and study the new set of modified conservation equations. Then in section IV, I shall show my results and finally I will discuss and summarize them.

\section{General Rankine-Hugoniot condition}

In this section I will discuss the general rankine-hugoniot condition as the conservation equations which balances the conversion of neutron proton ( $\mathrm{n}-\mathrm{p}$ ) matter to two-flavour quark matter, consisting of $u$ and d quarks along with electrons for ensuring charge neutrality. I heuristically assume the existence of a combustive phase transition front. Using the macroscopic conservation conditions, I examine the range of densities for which such a combustion front exists.

Let us consider the physical situation where a combustion front has been generated in the core of the Neutron star. This front propagates outwards through the neutron star with a certain velocity, leaving behind a u-d-e matter. In the following, I denote all the physical quantities in the hadronic sector by subscript 1 and those in the quark sector by subscript 2 . The conservation condition for energy-momentum and baryon number relates the quantities on the opposite sides of the front. In the rest frame of the combustion front, these conservation conditions is given by [24, 26, 27]:

$$
\begin{gathered}
\omega_{1} v_{1}^{2} \gamma_{1}^{2}+p_{1}=\omega_{2} v_{2}^{2} \gamma_{2}^{2}+p_{2} \\
\omega_{1} v_{1} \gamma_{1}^{2}=\omega_{2} v_{2} \gamma_{2}^{2}
\end{gathered}
$$

and

$$
n_{1} v_{1} \gamma_{1}=n_{2} v_{2} \gamma_{2}
$$

In the above three conditions $v_{i}(\mathrm{i}=1,2)$ is the velocity, $p_{i}$ is the pressure, $\gamma_{i}=\frac{1}{\sqrt{1-v_{i}^{2}}}$ is the Lorentz factor, $\omega_{i}=\epsilon_{i}+p_{i}$ is the specific enthalpy and $\epsilon_{i}$ is the energy density of the respective phases.

The velocities of the matter in the two phases, given by equations (1-3), can be solved, such that [26]:

$$
v_{1}^{2}=\frac{\left(p_{2}-p_{1}\right)\left(\epsilon_{2}+p_{1}\right)}{\left(\epsilon_{2}-\epsilon_{1}\right)\left(\epsilon_{1}+p_{2}\right)}
$$


and

$$
v_{2}^{2}=\frac{\left(p_{2}-p_{1}\right)\left(\epsilon_{1}+p_{2}\right)}{\left(\epsilon_{2}-\epsilon_{1}\right)\left(\epsilon_{2}+p_{1}\right)} .
$$

It is possible to classify the various conversion mechanisms by comparing the velocities of the respective phases with the corresponding velocities of sound, denoted by $c_{s i}$, in these phases. Thes conditions are summarized in [28].

For the conversion to be physically possible, velocities should satisfy an additional condition, namely, $0 \leq v_{i}^{2} \leq 1$. Here I find that the velocity condition puts severe constraint on the allowed equations of state.

\section{Magnetic field Inclusion}

In a conducting gas, a magnetic field can interact strongly with the flow. The analysis of the shock waves therefore becomes more complex, but the basic principles remains the same. A set of jump condition can again be derived, but they are considerably more complicated than the pure hydrodynamic shock case. The extra complexity arises both from the presence of extra variable, namely the magnetic field strength, and also from the fact that the magnetic field and the matter velocities may be inclined with the shock normal.

A shock propagating through an magnetic fluid produces a significant difference in matter properties on either side of the shock front. The thickness of the front is determined by a balance between convective and dissipative effects. However, dissipative effects at high temperature are only comparable to convective effects when the spatial gradients in matter variables become extremely large. Hence, shocks in such matter tend to be extremely narrow, and are well-approximated as discontinuity. The hydrodynamical equations, and Maxwell's equations, can be integrated across a shock to give a set of jump conditions which relate matter properties on each side of the shock front. If the shock is sufficiently narrow then these relations become independent of its detailed structure.

In the rest frame of the shock, the shock front coincides with the $y-z$ plane. Furthermore, the regions of the plasma upstream and downstream of the shock, which are termed regions 1 and 2, respectively, be spatially uniform and non-time-varying. It follows that $\partial / \partial t=\partial / \partial y=\partial / \partial z=0$. Moreover, $\partial / \partial x=0$, except in the immediate vicinity of the shock. Finally, let the velocity and magnetic fields upstream and downstream of the shock 
all lie in the $x-y$ plane. The magnetic field is given by $B_{i}$ for the respective phases.

The first nontrivial shock is called perpendicular shock in which both the upstream and downstream plasma flows are perpendicular to the magnetic field, as well as the shock front. The conservation condition are given by

$$
\begin{aligned}
\omega_{1} v_{1}^{2} \gamma_{1}^{2}+p_{1}+\frac{B_{1}^{2}}{8 \pi} & =\omega_{2} v_{2}^{2} \gamma_{2}^{2}+p_{2}+\frac{B_{2}^{2}}{8 \pi} \\
\omega_{1} v_{1} \gamma_{1}^{2}+v_{1} \gamma_{1} \frac{B_{1}{ }^{2}}{4 \pi} & =\omega_{2} v_{2} \gamma_{2}^{2}+v_{2} \gamma_{2} \frac{B_{2}^{2}}{4 \pi} \\
B_{1} v_{1} \gamma_{1} & =B_{2} v_{2} \gamma_{2}
\end{aligned}
$$

and

$$
n_{1} v_{1} \gamma_{1}=n_{2} v_{2} \gamma_{2}
$$

The first three equation can be reduced to two equation, given by

$$
\begin{aligned}
& \omega_{1} v_{1}^{2} \gamma_{1}^{2}+p_{1}+\frac{B_{1}^{2}}{8 \pi}=\omega_{2} v_{2}^{2} \gamma_{2}^{2}+p_{2}+\frac{B_{1}^{2}}{8 \pi}\left(\frac{v_{1} \gamma_{1}}{v_{2} \gamma_{2}}\right)^{2} \\
& \omega_{1} v_{1} \gamma_{1}^{2}+v_{1} \gamma_{1} \frac{B_{1}^{2}}{4 \pi}=\omega_{2} v_{2} \gamma_{2}^{2}+v_{2} \gamma_{2} \frac{B_{1}^{2}}{4 \pi} \frac{\left(v_{1} \gamma_{1}\right)^{2}}{v_{2} \gamma_{2}}
\end{aligned}
$$

I now solve for $v_{1}$ nad $v_{2}$.

The most general shock is the oblique shock in which the plasma velocities and the magnetic fields on each side of the shock are neither parallel nor perpendicular to the shock front. The Rankine-Hugoniot condition are given by

$$
\begin{aligned}
\omega_{1} v_{1 x}^{2} \gamma_{1}^{2}+p_{1}+\frac{B_{1}^{2}}{8 \pi}-\frac{B_{1 x}{ }^{2}}{4 \pi} & =\omega_{2} v_{2 x}^{2} \gamma_{2}^{2}+p_{2}+\frac{B_{2}^{2}}{8 \pi}-\frac{B_{2 x}{ }^{2}}{4 \pi} \\
\omega_{1} v_{1 x} v_{1 y} \gamma_{1}^{2}+v_{1 x} \gamma_{1} \frac{B_{1 x} B_{1 y}}{4 \pi} & =\omega_{2} v_{2 x} v_{2 y} \gamma_{2}^{2}+v_{2 x} \gamma_{2} \frac{B_{2 x} B_{2 y}}{4 \pi} \\
\omega_{1} v_{1 x} \gamma_{1}^{2}+v_{1 x} \gamma_{1} \frac{B_{1}{ }^{2}}{4 \pi}-\frac{B_{1 x}\left(B_{1} \cdot v_{1}\right) \gamma_{1}}{4 \pi} & =\omega_{2} v_{2 x} \gamma_{2}^{2}+v_{2 x} \gamma_{2} \frac{B_{2}{ }^{2}}{4 \pi}-\frac{B_{2 x}\left(B_{2} \cdot v_{2}\right) \gamma_{2}}{4 \pi}
\end{aligned}
$$




$$
\begin{aligned}
B_{1 y} v_{1 x} \gamma_{1}-v_{1 y} B_{1 x} \gamma_{1} & =B_{2 y} v_{2 x} \gamma_{2}-v_{2 y} B_{2 x} \gamma_{2} \\
B_{1 x} & =B_{2 x}
\end{aligned}
$$

and

$$
n_{1} v_{1 x} \gamma_{1}=n_{2} v_{2 x} \gamma_{2}
$$

There may be two easier cases for the above complicate equation.

Case 1. $v_{1 y}=0=v_{2 y}$

Then the first four equation would simplify to

$$
\begin{gathered}
\omega_{1} v_{1 x}^{2} \gamma_{1}^{2}+p_{1}+\frac{B_{1}{ }^{2}}{8 \pi}=\omega_{2} v_{2 x}^{2} \gamma_{2}^{2}+p_{2}+\frac{B_{2}{ }^{2}}{8 \pi} \\
\omega_{1} v_{1} \gamma_{1}^{2}+v_{1} \gamma_{1} \frac{B_{1}^{2}}{8 \pi}-\frac{B_{1 x}\left(B_{1} \cdot v_{1}\right) \gamma_{1}}{4 \pi}=\omega_{2} v_{2} \gamma_{2}^{2}+v_{2} \gamma_{2} \frac{B_{2}{ }^{2}}{8 \pi}-\frac{B_{1 x}\left(B_{2} \cdot v_{2}\right) \gamma_{2}}{4 \pi} \\
B_{2 y}=\frac{v_{1} \gamma_{1}}{v_{2} \gamma_{2}} B_{1 y}
\end{gathered}
$$

Case 2. $B_{1 y}=0=B_{2 y}$

$$
\begin{aligned}
\omega_{1} v_{1 x}^{2} \gamma_{1}^{2}+p_{1} & =\omega_{2} v_{2 x}^{2} \gamma_{2}^{2}+p_{2}, \\
\omega_{1} v_{1 x} \gamma_{1}^{2}+v_{1 x} \gamma_{1} \frac{B_{1}{ }^{2}}{8 \pi}-\frac{B_{1 x}\left(B_{1} \cdot v_{1}\right) \gamma_{1}}{4 \pi} & =\omega_{2} v_{2 x} \gamma_{2}^{2}+v_{2 x} \gamma_{2} \frac{B_{2}{ }^{2}}{8 \pi}-\frac{B_{1 x}\left(B_{2} \cdot v_{2}\right) \gamma_{2}}{4 \pi} \\
\omega_{1} v_{1 x} v_{1 y} \gamma_{1}{ }^{2} & =\omega_{2} v_{2 x} v_{2 y} \gamma_{2}{ }^{2}
\end{aligned}
$$

\section{Results}

I start my calculations by using the nuclear matter EOS obtained through nonlinear Walecka model [29]. In the present paper, I consider the conversion of nuclear matter, consisting of only nucleons, to a two-flavour quark matter. The final composition of the quark matter is determined from the 
nuclear matter EOS by enforcing the baryon number conservation during the conversion process. While describing the state of matter for the quark phase I consider a range of values for the bag constant. Nuclear matter EOS is calculated at zero temperature, whereas, the two-flavour quark matter EOS is obtained both at zero temperature as well as at finite temperatures as during the propagation of the shock it may heat up the matter.

To examine the nature of the hydrodynamical front, arising from the neutron to two-flavour quark matter conversion, I plot, in fig.1, the quantities $v_{1}, v_{2}, c_{s 1}$ and $c_{s 2}$ as functions of the baryon number density $\left(n_{B}\right)$. As mentioned earlier, the $\mathrm{u}$ and $\mathrm{d}$ quark content in the quark phase is kept same as the one corresponding to the quark content of the nucleons in the hadronic phase. With these fixed densities of the massless $u$ and $d$ quarks and electrons, the EOS of the two-flavour matter has been evaluated using the bag model prescription. I find that the velocity condition $\left(v_{i}^{2}>0\right)$ is satisfied only for a small window of $\approx \pm 5.0 \mathrm{MeV}$ around the bag pressure $B^{1 / 4}=160 \mathrm{MeV}$. The constraint imposed by the above conditions results in the possibility of deflagration, detonation or supersonic front as shown in the fig. 1.

In fig. 1, I considered both the phases to be at zero temperature. A possibility, however, exist that a part of the internal energy is converted to heat energy, thereby increasing the temperature of the two-flavour quark matter during the exothermic combustive conversion process. In fig. 2, I plot the variation of velocities with density at $T=50 \mathrm{MeV}$, for which significant change is noticed. This figure show that the range of values of baryon density, for which the flow velocities are physical, increases with temperature. In the present paper I have considered only the zero temperature nuclear matter EOS. On the other hand, equation of state of quark matter has a finite temperature dependence and hence the difference between $v_{1}$ and $v_{2}$, varies with temperature.

The variation of the velocities with temperature is due to the fact that higher temperature means higher energy. As the energy increases the particles becomes more energetic which means the matter becomes more excited and compressible. As matter becomes more compressible due to increase in temperature, now there is a chance for shock formation which was previously not possible.

Till now I have used equation for the normal hydrodynamics for the generation of the curves. The change in temperature in the EOS accounted for the change in the nature of the graph. Now I will plot graphs solving mag- 


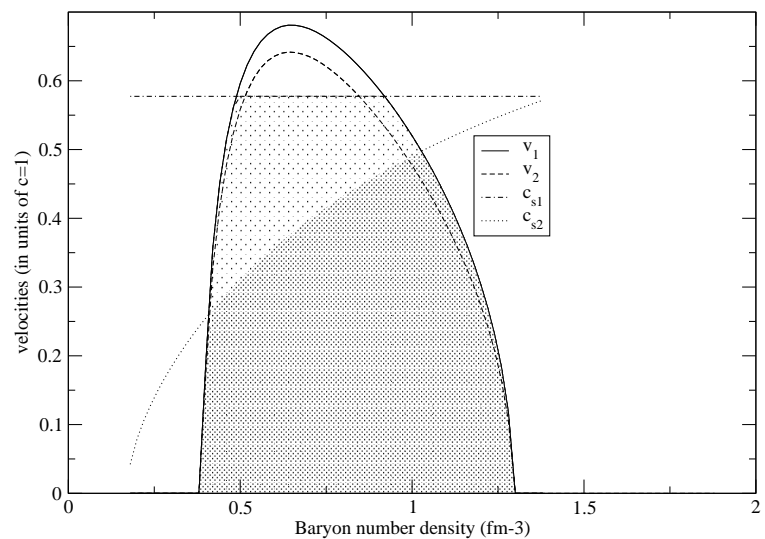

Figure 1: Variation of different velocities with baryon number density for $\mathrm{T}=0 \mathrm{MeV}$, $B^{1 / 4}=160 \mathrm{MeV}$ and strange quark mass $m_{s}=200 \mathrm{MeV}$. The dark-shaded region correspond to deflagration, light-shaded region correspond to detonation and the unshaded region correspond to supersonic conversion processes.

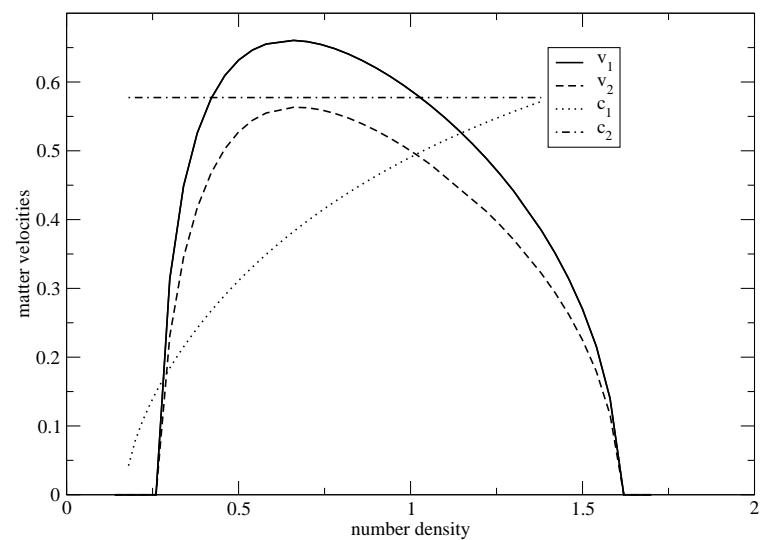

Figure 2: Variation of velocities with baryon number density for $\mathrm{T}=80 \mathrm{MeV} B^{1 / 4}=$ $160 \mathrm{MeV}$ and strange quark mass $m_{s}=200 \mathrm{MeV}$. Different regions correspond to different modes of conversion similar to that of fig 1 . 
netic field induced hydrodynamics. I solve the new conservation conditions for the perpendicular shock in which both the upstream and downstream plasma flows are perpendicular to the magnetic field, as well as the shock front.

In fig. 3 I have plotted curves for different velocities with baryon density for the zero temperature case. The magnetic field used for the generation of the curve is $B=5 \times 10^{15} \mathrm{G}$. I find that due to introduction of the magnetic field the range of values of baryon density, for which the flow velocities are physical, increases. Its nature is quite similar to that for the case of temperature. Due to the introduction of the magnetic field the the pressure increases (pressure due to magnetic field is $B^{2} / 8 \pi$ ) for the same value of baryon number density. The magnetic field in the respective phases adjust in such a way that the resultant energy and pressure of the both phases gets modified to make the range of baryon number density to increase. For both higher and lower values of baryon density, previously there was less chance of shock formation, but now due to the introduction of the magnetic field there is a greater chance of shock formation. And therefore the range of baryon density gets much wider.

Next I plot curves for different velocities for the finite temperature case $T=80 \mathrm{MeV}$. For same value of the magnetic field $B=5 \times 10^{15} \mathrm{G}$, the range of baryon density gets much more wider. This is due to the fact that now both the temperature and the magnetic field work hand by hand to increase the chances of shock generation. Both process ensures by its own way that the matter parameters adjust itself in such a way that there is a greater probability of shock generation.

In fig. 5 I have plotted exclusively $v_{1}$ and $v_{2}$ for two cases, one without magnetic field and the other with magnetic field. The nature of the curve remains same, that is $v_{1}$ is always greater than $v_{2}$, which means the shock front propagates outward of the star. The range of baryon density, for which the matter velocities are finite, increases with magnetic field. I have plotted this for the zero temperature case. In fig. 6 I have plotted the same for the finite temperature case and find that for lesser value of magnetic field, same increase in range of baryon number density is seen. All the reasons for this nature is explained in the previous paragraph.

In fig 7. I have plotted $v_{1}$ for different values of magnetic field. I find that as the value of the magnetic field increases the range of baryon number density increases which is what was expected (and discussed previously). But as we go on increasing the magnetic field $v_{1}$ does not comes down on 


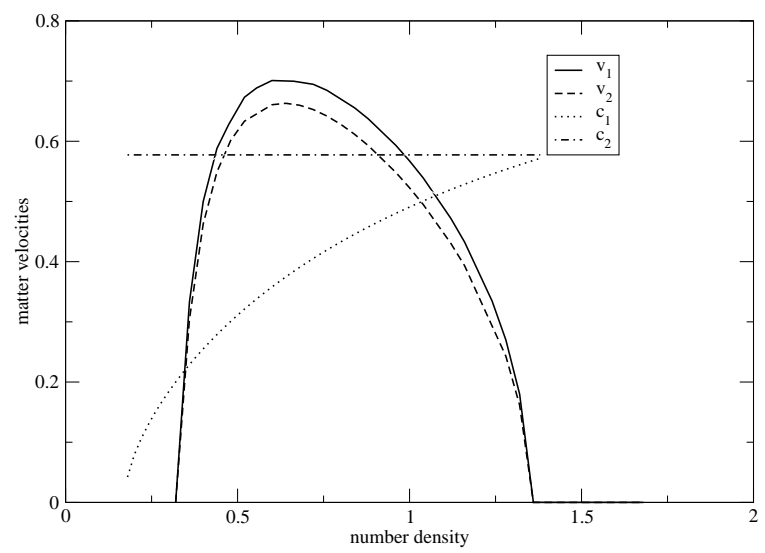

Figure 3: Variation of different flow velocities with baryon number density for $\mathrm{T}=0 \mathrm{MeV}$ and magnetic field of $B=5 \times 10^{15} G$.

the lower half of the curve. This is due to the fact that, at such high value of the magnetic field the matter becomes unstable. At other half where matter is at much higher density, it can support such field strength. But, if we further increase the magnetic field the matter cannot support such high fields whatever the density might be. The maximum value of magnetic field that matter can support is few times $10^{17} G$ (in our case the cut off value is $2 \times 10^{17} G$ ). So I find that there is a cut off value for the magnetic field, and from here we can indirectly say that NS also can support up to a finite value of magnetic field. This curve is plotted for zero temperature, and in fig. 8 I have plotted the same for finite temperature. Qualitatively the nature of the graph remains the same only the quantitative value of the magnetic field changes. It shows that hotter matter can support lesser value of magnetic field than the colder one.

\section{Summary and discussion}

Finally in this section I summarize my results. I find that rankinehugoniot condition can be solved to determine the condition for different types of wave generation in a neutron star. It also determines the mode of the propagation of the wave front. The temperature (finite temperature of the matter) helps in the generation of the front and as the temperature rises the wave front can generate both at much lower and at much higher baryon 


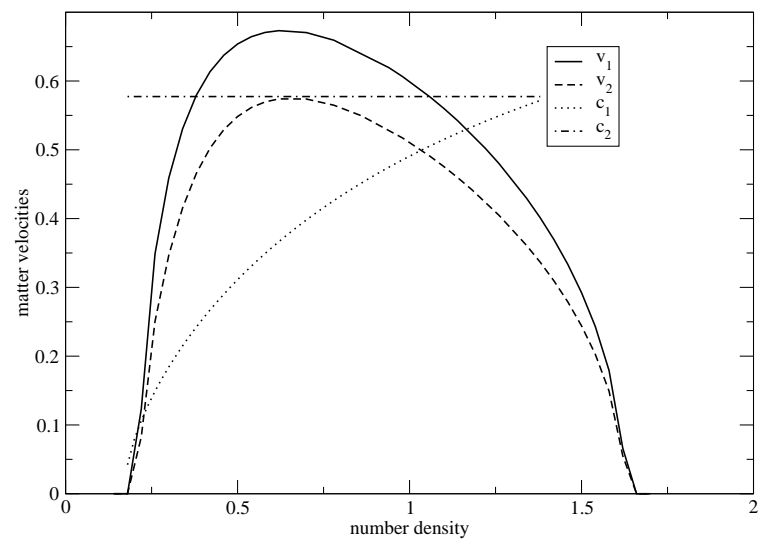

Figure 4: Variation of velocities with baryon number density for $T=80 \mathrm{MeV}$, and magnetic field of $B=5 \times 10^{15} G$.

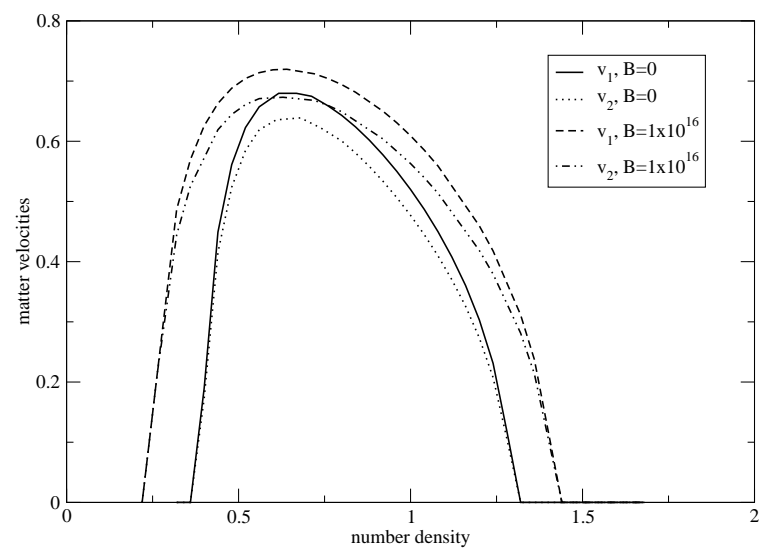

Figure 5: Comparison of $v_{n}$ and $v_{s}$ with baryon number density for $T=0 \mathrm{MeV}$ for two values of magnetic field strength $B=0 G$ and $B=1 \times 10^{16} G$. 


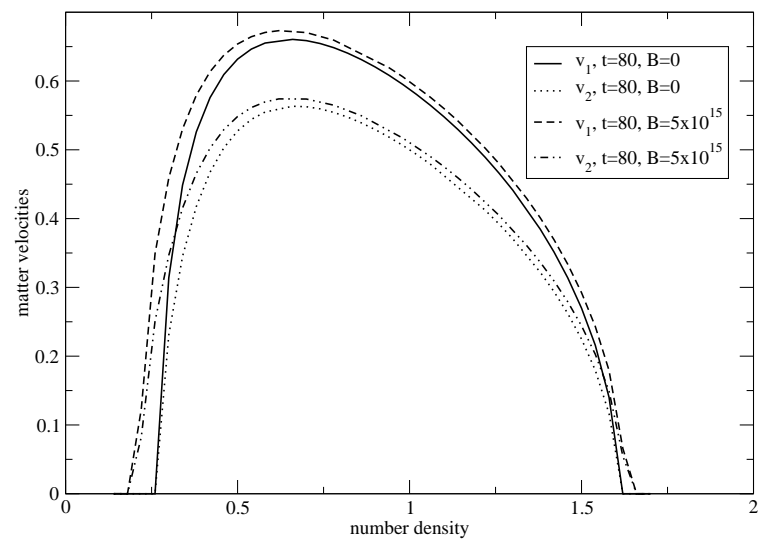

Figure 6: Comparison of $v_{n}$ and $v_{s}$ with baryon number density for $T=80 \mathrm{MeV}$ for two values of magnetic field strength $B=0 G$ and $B=5 \times 10^{15} G$.

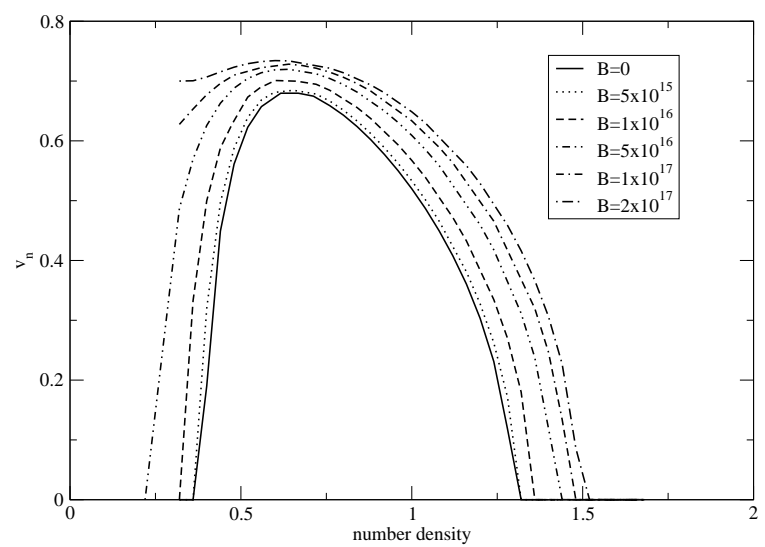

Figure 7: Comparison of $v_{n}$ with baryon number density for $T=0 \mathrm{MeV}$ for different values of magnetic field strength. 


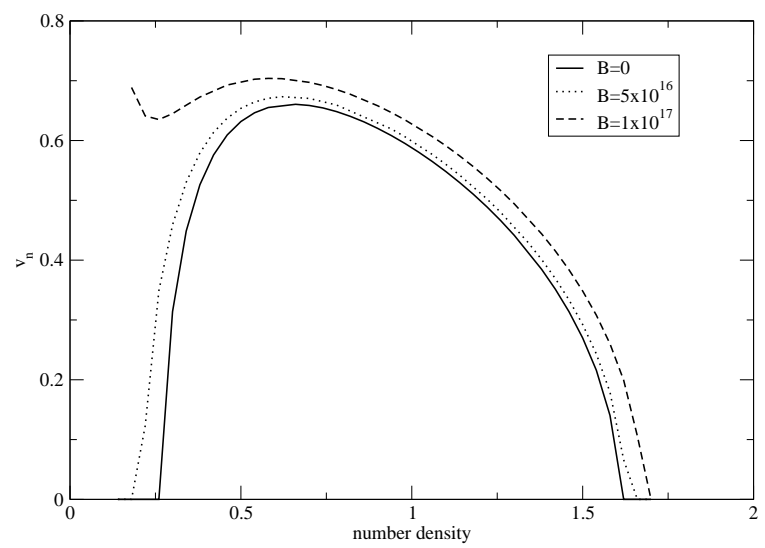

Figure 8: Comparison of $v_{n}$ with baryon number density for $T=80 \mathrm{MeV}$ for different values of magnetic field strength.

densities which was not possible for the zero temperature case. Next I write down the modified conservation conditions in presence of magnetic field. I have written down conditions for both the perpendicular and oblique shock waves. The inclusion of the magnetic field introduces not only extra conditions but also the earlier existing conditions gets modified. I have solved the velocity of the matter of the two phases and plotted curves for the perpendicular wave, which was not obtained analytically. The conditions cannot be solved analytically and and therefore I have solved the nonlinear simultaneously equation numerically. I have matched my nonmagnetic numerical results with the analytically solvable nonmagnetic solutions. The oblique wave equation gets very much complicated and the simultaneous equations does not converges for different value of the baryon densities. So I have not plotted the results of the oblique waves.

Solving the perpendicular wave for finite magnetic field I find that the range of baryon density, for which the flow velocities of matter are physical, increases with magnetic field strength. This is because by the introduction of the magnetic field the resultant pressure and energy redistribute in such a way that, for same baryon density, the changes for shock generation increases. I also find that there is a cut off magnetic field strength that matter can support, beyond which the matter becomes unstable and the flow velocities becomes imaginary. This on the other hand suggest that a NS cannot support 
magnetic field beyond a certain field strength. To finally summarize our result I mention that this is the first instance where such a treatment of modified conservation condition has been done in the presence of magnetic field in the astrophysical phase transition scenario. This provides with new interesting results, that has not been anticipated before and also indirectly hints at the instability of the NS at very high magnetic field. More interesting results is anticipated if the full oblique wave equations can be solved and I am now focussing mainly on that problem.

I would like to thank Grant No. SR/S2HEP12/2007, funded by DST, India for financial support.

\section{References}

[1] A. H. Taub, Physical Review, 74, 328 (1948)

[2] K. S. Thorne, Astrophys. J., 179, 897 (1973)

[3] A. Lichnerowicz, 1967, Relativistic hydrodynamics and Magnetohydrodynamics, New York, Benjamin

[4] P. J. Steinhardt, Phys. Rev. D, 25, 2074 (1982)

[5] J. Cleymans, R. V. Gavai, E Suhonen, Physics reports, 130, 217 (1986)

[6] K. A. Van Riper, Astrophys. J., 232, 558 (1979)

[7] E. Witten, Phys. Rev. D30, 272 (1984)

[8] E. Farhi and R. L. Jaffe, Phys. Rev. D30, 2379 (1984)

[9] A. Bhattacharyya, S. K. Ghosh, M. Hanauske and S. Raha, Phys. Rev. C71, 048801 (2005)

[10] A. Bhattacharyya, S. K. Ghosh and S. Raha, Phys. Lett. B635, 195 (2006)

[11] A. Bhattacharyya and S. K. Ghosh, Mod. Phys. Lett. A22, 1019 (2007)

[12] C. Alcock, E. Farhi and A. Olinto, Astrophys. J. 310, 261 (1986)

[13] N. K. Glendenning, Nucl. Phys. (Proc. Suppl.) B24, 110 (1991); Phys. Rev. D46, 1274 (1992) 
[14] A. Olinto, Phys. Lett. B192, 71 (1987); Nucl. Phys. (Proc. Suppl.) B24, $103(1991)$

[15] M. L.Olesen and J. Madsen, Nucl. Phys. (Proc. Suppl.) B24, 170 (1991)

[16] H. Heiselberg, G. Baym and C. J. Pethick, Nucl. Phys. (Proc. Suppl.) B24, 144 (1991)

[17] G. Lugones, O. G. Benvenuto and H. Vucetich, Phys. Rev. D50, 6100 (1994)

[18] O. G. Benvenuto, and J. E. Horvarth, Phys. Lett. B213, 516 (1988)

[19] O. G. Benvenuto, J. E. Horvarth and H. Vucetich, Int. J. Mod. Phys. A4, 257 (1989); O. G. Benvenuto and J. E. Horvarth, Phys. Rev. Lett. 63, 716 (1989)

[20] H. T. Cho, K. W. Ng and A. W. Speliotopoulos, Phys. Lett. B326, 111 (1994)

[21] I. Tokareva, A. Nusser, V. Gurovich and V. Folomeev, Int. J. Mod. Phys. D14, 33 (2005)

[22] Z. Berezhiani, I. Bombaci, A. Drago, F. Frontera and A. Lavagno, Astrophys. J. 586, 1250 (2003)

[23] I. Bombaci, I. Parenti and I. Vidana, Astrophys. J. 614, 314 (2004)

[24] Tokareva, I., Nusser, A., Gurovich, V., Folomeev, V., 2005, Int. J. Mod. Phys., D14, 33

[25] A. Bhattacharyya, S. K. Ghosh, P. Joarder, R. Mallick and S. Raha, Phys. Rev. C74, 065804 (2006)

[26] Landau, L. D., and Lifshitz, E. M., 1987, Fluid Mechanics, Pergamon Press, New York

[27] Anile, A. M., 1989, Relativistic fluids and Magneto-fluids : with application in Astrophysics and Plasma Physics, Cambridge University Press, U.K.

[28] Laine, M., 1994, Phys. Rev., D49, 3847

[29] J. Ellis, J. I. Kapusta and K. A. Olive, Nucl. Phys. B, 348, 345 (1991) 